Bio - grafía. Escritos sobre la Biología y su Enseñanza. ISSN 2027-1034

Edición Extraordinaria. p.p. 1360 - 1368

Memorias del IX Encuentro Nacional de Experiencias en Enseñanza de la Biología y la Educación Ambiental. IV Congreso Nacional de Investigación en Enseñanza de la Biología.

\title{
LA TEMÁTICA SALUD Y EDUCACIÓN EN SALUD, EN LAS PUBLICACIONES SOBRE FORMACIÓN DE PROFESORES DE BIOLOGÍA
}

\section{THE THEMATIC HEALTH AND HEALTH EDUCATION, PUBLICATIONS ON THE TRAINING OF TEACHERS OF BIOLOGY}

\author{
Eliane Gonçalves dos Santos ${ }^{1}$ \\ Maria Cristina Pansera de Araújo ${ }^{2}$
}

\section{RESUMEN:}

El conocimiento sobre la Educación en Salud (ES) en la formación de Profesores de Biología, en tesis y disertaciones publicadas entre 2006 a 2016, fue analizado para identificar, reflexionar y discutir los enfoques propuestos. La metodología de trabajo consistió en el análisis de los documentos seleccionados con los descriptores salud, educación en salud en la formación de profesores de biología, en el título, palabras clave o resumen. Se identificaron veintiséis trabajos entre tesis y disertaciones. El análisis permitió reconocer seis categorías: Educación y Sexualidad; Educación en Salud y el Proceso de Formación de Profesores; Salud y Educación; Educación y Hábitos Alimenticios; Educación en Salud en los Libros Didácticos; Educación y Promoción de la Salud. Se identificó la preocupación de la comunidad académica en entender y desarrollar acciones y prácticas pedagógicas que estimulen un aumento del entendimiento de la ES, en la perspectiva de la promoción de la vida de calidad, en la escuela. Pocas publicaciones investigaron la formación inicial y continuada, lo que abre la posibilidad de nuevos estudios.

PALABR AS CLAVE: Educación para la Salud. Procesos de Formación. Producción Científica.

ABSTRACT: The knowledge about Health Education (ES) in the training of Biology Teachers, in theses and dissertations published between 2006 and 2016, was analyzed to recognize, reflect and question the proposed approaches. The work methodology consisted of the analysis of the selected documents with the descriptors health, health education in the training of biology teachers, in the title, keywords or abstract. Twenty-six papers were identified between theses and dissertations. The analysis allowed to recognize six categories: Education and Sexuality; Health Education and the Formative Process of Teachers; Health and Education; Education and Eating Habits; Health Education in Textbooks; Education and Health Promotion. It

\footnotetext{
${ }^{1}$ Doutoranda no Programa de Pós-Graduação em Educação nas Ciências da Universidade Regional do Noroeste do Estado do Rio grande do Sul - Unijuí, RS - Brasil. Professora do curso de Ciências Biológic as - Licenciatura da Universidade Federal da Fronteira Sul - UFFS, campus Cerro Largo, RS - Brasil. Email: elianesan@bol.com.br

${ }^{2}$ Professora Titular do Programa de Pós-Graduação em Educação nas Ciências da Universidade Regional do Noroeste do Estado do Rio grande do Sul - Unijuí, RS - Brasil. Email: pansera@unijui.edu.br
} 
Bio - grafía. Escritos sobre la Biología y su Enseñanza. ISSN 2027-1034

Edición Extraordinaria. p.p. 1360 - 1368

Memorias del IX Encuentro Nacional de Experiencias en Enseñanza de la Biología y la Educación Ambiental. IV Congreso Nacional de Investigación en Enseñanza de la Biología.

was identified the concern of the academic community to understand and develop pedagogical actions and practices that promote the extension of the ES understanding, with a view to promoting quality life in school. Few publications have addressed initial and continuing training, which raises new studies.

KEYSWORDS: Health Education. Formative processes. Scientific Production

\section{INTRODUÇÃO}

Muito é discutido na literatura e eventos da Educação sobre um processo formativo que contemple novas demandas da sociedade e superem o ensino tradicional ancorado na oratória do professor e nos livros didáticos. Entre os temas, a Educação em Saúde constitui desafio, mesmo que as discussões, em âmbito escolar, não sejam recentes, visto que desde o início do século $X X$ a temática Saúde está inserida nos currículos escolares (Monteiro \& Bizzo, 2015). Na escola do século XXI, os professores formadores necessitam repensar seu fazer e compreender que, somente, quando suas práticas pedagógicas forem significadas pelos acadêmicos, articuladas a teoria, haverá avanço no ensino.

Mohr, em 2002, na sua tese "A natureza da Educação em Saúde no Ensino Fundamental e os Professores de Ciências" analisou a questão, constituindo-se referência na área de ensino.

$\mathrm{Na}$ atualidade, a preocupação dos pesquisadores da área de Educação recai sobre um ensino que trate a saúde numa abordagem sistêmica e possibilite aos sujeitos mudanças de comportamento e de estilos de vida. Nessa perspectiva, os contextos social e cultural são elementos importantes no processo da Educação em Saúde (ES), principalmente no que se refere a formação de professores. Conforme Carvalho \& Jourdan, a abordagem da ES na escola é tradicional com transmissão de informações insuficientes, por isso necessitam outras tematizações ao:

[...] proporcionar aos alunos a aquisição de conhecimentos e competências que lhes permita atuarem como cidadãos livres e responsáveis no campo da saúde. Isso implica serem capazes de compreender não só o impacte dos determinantes de saúde, mas também da necessidade de mudarem para estilos de vida mais saudáveis e criarem melhores condições de vida, a fim de melhorar a sua saúde pessoal e comunitária (Carvalho \& Jourdan, 2014, p. 104).

A desconstrução do entendimento de saúde como ausência de doença exige outra organização curricular e formação de professores de modo a superar esta visão, na construção de diálogos provocadores de mudanças no currículo, em que a Saúde é apresentada de modo sistêmico e o sujeito em sua integralidade. Nessa perspectiva, temos como objetivo analisar as teses e dissertações publicadas entre 2006 a 2016 sobre Saúde e Educação em Saúde na formação de professores, para reconhecer, refletir e questionar as abordagens propostas para reconhecer as principais abordagens, refletir e sintetizar os estudos realizados sobre o tema em questão. 
Bio - grafía. Escritos sobre la Biología y su Enseñanza. ISSN 2027-1034

Edición Extraordinaria. p.p. 1360 - 1368

Memorias del IX Encuentro Nacional de Experiencias en Enseñanza de la Biología y la Educación Ambiental. IV Congreso Nacional de Investigación en Enseñanza de la Biología.

\section{METODOLOGIA:}

É uma pesquisa qualitativa do tipo documental que "pode se constituir numa técnica valiosa de abordagem de dados qualitativos" (Lüdke \& André, 2013, p. 45). Para estabelecer o estado do conhecimento sobre Saúde e Educação em Saúde foi realizada uma busca no Portal de Teses e Dissertações da Coordenação de Aperfeiçoamento de Pessoal de Nível Superior (CAPES), com recorte temporal de 2006 a 2016. Os descritores saúde, educação em saúde e formação de professores de biologia orientaram a busca no título, palavras-chave ou resumo, e permitiram identificar vinte e seis trabalhos entre teses e dissertações (Quadro 1). A busca e análise fundamentaram-se em Morosini (2015, p. 102), que define estado de conhecimento como a "identificação, registro, categorização que levam à reflexão e síntese sobre a produção científica de uma determinada área, em um determinado espaço de tempo, congregando periódicos, teses, dissertações e livros sobre uma temática específica".

\section{Quadro 1. Teses e dissertações com a temática Educação em Saúde no Ensino, publicadas de 2006 a 2016.}

\begin{tabular}{|c|c|c|c|c|}
\hline $\mathbf{N}^{\circ}$ & Título & Autor & Ano & $\begin{array}{c}\text { Tese (T), } \\
\text { Dissertação (D) } \\
\text { e Instituição }\end{array}$ \\
\hline P01 & $\begin{array}{l}\text { Formação de sanitaristas: } \\
\text { cartografias de uma pedagogia da } \\
\text { educação em saúde coletiva }\end{array}$ & $\begin{array}{l}\text { Armani, Teresa } \\
\text { Borgert }\end{array}$ & 2006 & $\begin{array}{l}\text { D - Universidade } \\
\text { Federal do Rio } \\
\text { Grande do Sul - } \\
\text { UFRGS }\end{array}$ \\
\hline P02 & $\begin{array}{l}\text { Sexualidade na adolescência: } \\
\text { trabalhando a Pesquisa-Ação com } \\
\text { referenciais teórico-metodológicos } \\
\text { de Paulo Freire }\end{array}$ & $\begin{array}{ll}\text { Pereira, } & \text { Kely } \\
\text { Cristina } & \end{array}$ & 2007 & $\begin{array}{l}\text { D - Universidade } \\
\text { de São Paulo - } \\
\text { USP }\end{array}$ \\
\hline P03 & $\begin{array}{l}\text { Educação, Empowerment no } \\
\text { Campo da Promoção da Saúde: } \\
\text { revisão da literatura brasileira no } \\
\text { período de } 1997 \text { a } 2008\end{array}$ & $\begin{array}{l}\text { Pereira, Mônica } \\
\text { Rodrigues } \\
\text { Saraiva }\end{array}$ & 2009 & $\begin{array}{lr}\text { D } & \text { Escola } \\
\text { Nacional } & \text { de } \\
\text { Saúde } & \text { Pública } \\
\text { Sérgio Arouca/RJ }\end{array}$ \\
\hline P04 & $\begin{array}{llr}\text { Educação e } & \text { saúde } & \text { das } \\
\text { adolescentes na periferia } & \text { urbana: } \\
\text { estudo de caso em } & \text { Novo } \\
\text { Hamburgo - RS }\end{array}$ & $\begin{array}{l}\text { Chamis, Niva } \\
\text { Maria Almeida }\end{array}$ & 2010 & D - UFRGS \\
\hline P05 & $\begin{array}{lcr}\text { Educação } & \text { Sexual, } & \text { Corpo } \\
\text { Sexualidade } & \text { na visão dos alunos e } \\
\text { professores } & \text { no } & \text { Ensino } \\
\text { Fundamental } & & \end{array}$ & $\begin{array}{l}\text { Moizés, Julieta } \\
\text { Seixas }\end{array}$ & 2010 & $\mathrm{~T}-\mathrm{USP}$ \\
\hline P06 & $\begin{array}{l}\text { Representação Social de Saúde, } \\
\text { Doença e Dengue para alunos do }\end{array}$ & $\begin{array}{l}\text { Szukala, } \\
\text { Claudia }\end{array}$ & 2010 & $\begin{array}{l}\text { D - Universidade } \\
\text { Católicar Dom } \\
\text { Bosco - UCDB/ }\end{array}$ \\
\hline
\end{tabular}


Bio - grafía. Escritos sobre la Biología y su Enseñanza. ISSN 2027-1034

Edición Extraordinaria. p.p. 1360 - 1368

Memorias del IX Encuentro Nacional de Experiencias en Enseñanza de la Biología y la Educación Ambiental. IV Congreso Nacional de Investigación en Enseñanza de la Biología.

\begin{tabular}{|c|c|c|c|c|}
\hline & Ensino Fundamental & Medeiros & & MS \\
\hline P07 & $\begin{array}{l}\text { Gênero e sexualidade } \text { na } \\
\text { educação de jovens e adultos: um } \\
\text { estudo de caso }\end{array}$ & $\begin{array}{l}\text { Oliveira, Karina } \\
\text { Fürstenau de }\end{array}$ & 2010 & D - UFRGS \\
\hline P08 & $\begin{array}{l}\text { A Temática Sexualidade no } \\
\text { Contexto Escolar: Diagnóstico } \\
\text { Situacional da Região Leste de } \\
\text { Goiânia, Goiás }\end{array}$ & $\begin{array}{l}\text { Chaveiro, Laine } \\
\text { Gomes }\end{array}$ & 2011 & $\begin{array}{l}\text { D - Universidade } \\
\text { Federal de } \\
\text { Goiás - UFG }\end{array}$ \\
\hline P09 & $\begin{array}{l}\text { Educação Sexual no Ensino de } \\
\text { Ciências: Um Estudo com Foco } \\
\text { nos Professores }\end{array}$ & $\begin{array}{l}\text { Silva, Otoniel } \\
\text { Alvaro da. }\end{array}$ & 2011 & $\begin{array}{l}\text { D - Universidade } \\
\text { Federal do } \\
\text { Paraná - UFPR }\end{array}$ \\
\hline $\mathrm{P} 10$ & $\begin{array}{l}\text { Alimentação Saudável na Escola: } \\
\text { Uma Construção Coletiva? }\end{array}$ & $\begin{array}{l}\text { Camozzi, Aida } \\
\text { Bruna Quilici }\end{array}$ & 2011 & $\bar{D}-\mathrm{UFG}$ \\
\hline P11 & $\begin{array}{l}\text { Tuberculose uma Conversa } \\
\text { Necessária entre Saúde, Ambiente } \\
\text { e Educação }\end{array}$ & $\begin{array}{l}\text { Donato Junior, } \\
\text { Giuseppe } \\
\text { Francesco } \\
\text { Antonio }\end{array}$ & 2011 & $\begin{array}{l}\text { D - Instituto Fed. } \\
\text { de Educ., Ciência } \\
\text { e Tecnol. do Rio } \\
\text { de } \\
\text { Janeiro/Nilópolis }\end{array}$ \\
\hline $\bar{P} 12$ & 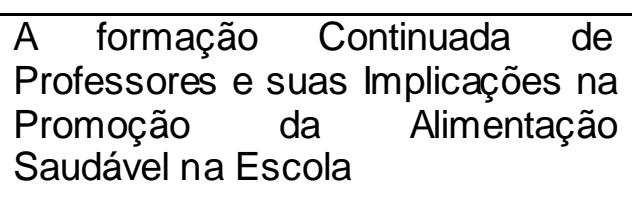 & $\begin{array}{l}\text { Oliveira, Miriam } \\
\text { Sampaio de }\end{array}$ & 2011 & $\begin{array}{l}\text { D Universidade } \\
\text { Federal de São } \\
\text { Paulo - Unifesp }\end{array}$ \\
\hline $\mathrm{P} 13$ & $\begin{array}{l}\text { Educação em Saúde: Avanços e } \\
\text { Dilemas na Trajetória de Formação } \\
\text { Contínua do Programa de } \\
\text { Educação em Saúde do Rio do } \\
\text { Sul, SC }\end{array}$ & Sebold, Rosita & 2011 & $\begin{array}{l}\text { D - Universidade } \\
\text { Regional de } \\
\text { Blumenau - } \\
\text { FURB }\end{array}$ \\
\hline$\overline{P 14}$ & 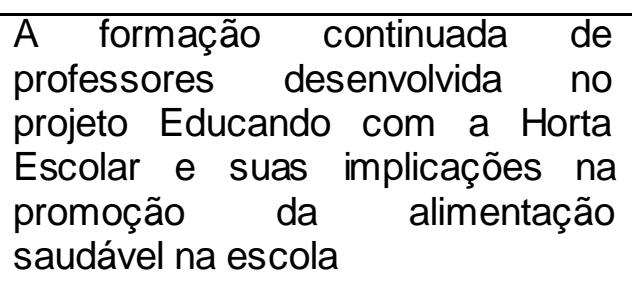 & $\begin{array}{l}\text { Oliveira, Miriam } \\
\text { Sampaio de }\end{array}$ & 2011 & $\begin{array}{l}\text { D - Universidade } \\
\text { Federal de São } \\
\text { Paulo - UNIFESP }\end{array}$ \\
\hline P15 & $\begin{array}{l}\text { A concepção de professores sobre } \\
\text { saúde na escola }\end{array}$ & $\begin{array}{lr}\text { Ferraro, } & \text { Maísa } \\
\text { Rezende } & \text { de } \\
\text { Melo } & \end{array}$ & 2011 & $D-U S P$ \\
\hline $\mathrm{P} 16$ & $\begin{array}{l}\text { As Imagens da Saúde em Livros } \\
\text { Didáticos de Ciências }\end{array}$ & $\begin{array}{lr}\text { Souza, } & \text { Lucia } \\
\text { Helena } & \text { Pralon } \\
\text { de } & \end{array}$ & 2011 & $\begin{array}{l}\text { T - Universidade } \\
\text { Federal do Rio de } \\
\text { Janeiro - UFRJ }\end{array}$ \\
\hline P17 & $\begin{array}{l}\text { O Aborto como um Problema de } \\
\text { Saúde Pública: Contribuições de }\end{array}$ & $\begin{array}{l}\text { Carpilovsky, } \\
\text { Cristiane Kohler }\end{array}$ & 2011 & T-UFRGS \\
\hline
\end{tabular}


Bio - grafía. Escritos sobre la Biología y su Enseñanza. ISSN 2027-1034

Edición Extraordinaria. p.p. 1360 - 1368

Memorias del IX Encuentro Nacional de Experiencias en Enseñanza de la Biología y la Educación Ambiental. IV Congreso Nacional de Investigación en Enseñanza de la Biología.

\begin{tabular}{|c|c|c|c|c|}
\hline & Saúde e Educação & & & \\
\hline P18 & $\begin{array}{l}\text { Educação Sexual na Escola } \\
\text { Pública como Estratégia de } \\
\text { Promoção da Saúde }\end{array}$ & $\begin{array}{l}\text { Brilhante, Aline } \\
\text { Veras Morais }\end{array}$ & 2012 & $\begin{array}{l}\text { D - Universidade } \\
\text { de Fortaleza - } \\
\text { Unifor }\end{array}$ \\
\hline P19 & $\begin{array}{l}\text { Educação em Saúde no Ensino } \\
\text { Fundamental: Uma Reflexão } \\
\text { acerca da Promoção da Saúde }\end{array}$ & $\begin{array}{l}\text { Damiani, Ana } \\
\text { Paula Macan }\end{array}$ & 2012 & $\begin{array}{l}\text { D - Universidade } \\
\text { do Extremo Sul } \\
\text { Catarinense - } \\
\text { UNESC }\end{array}$ \\
\hline P20 & $\begin{array}{l}\text { Educação em Saúde nas Escolas: } \\
\text { Elaboração de } \\
\text { Paradidático sobre doenças } \\
\text { viróticas Aids, Dengue e Gripe a } \\
\text { partir da Análise dos Livros } \\
\text { Didáticos de Biologia }\end{array}$ & $\begin{array}{l}\text { Barcelos, } \\
\text { Mariana } \quad \text { De } \\
\text { Oliveira }\end{array}$ & 2012 & $\mathrm{D}-\mathrm{PUC} / \mathrm{MG}$ \\
\hline P21 & 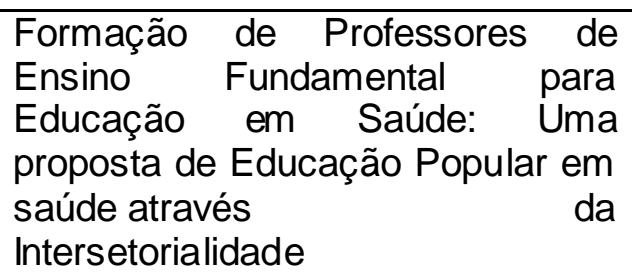 & $\begin{array}{l}\text { Pereira, Patricia } \\
\text { Carla Gandin }\end{array}$ & 2012 & $\begin{array}{l}\text { D - Universidade } \\
\text { do Vale do Itajaí }\end{array}$ \\
\hline P22 & $\begin{array}{l}\text { Uso de Textos de Divulgação } \\
\text { Científica como Estratégia de } \\
\text { Trabalho com Temas de Educação } \\
\text { em Saúde na Escola para } \\
\text { Educação de Jovens e Adultos } \\
\text { (EJA) }\end{array}$ & $\begin{array}{l}\text { Oliveira, Lidiane } \\
\text { Loiola de }\end{array}$ & 2013 & $\begin{array}{l}\text { D - Universidade } \\
\text { de Brasília - UNB }\end{array}$ \\
\hline P23 & $\begin{array}{l}\text { Os modos de estruturação da } \\
\text { educação em saúde na escola: das } \\
\text { concepções e do currículo às } \\
\text { práticas educativas e à } \\
\text { aprendizagem }\end{array}$ & $\begin{array}{l}\text { Marinho, Julio } \\
\text { Cesar Bresolin }\end{array}$ & 2013 & $\begin{array}{l}\text { D - Universidade } \\
\text { Federal do Rio } \\
\text { Grande - FURG }\end{array}$ \\
\hline P24 & 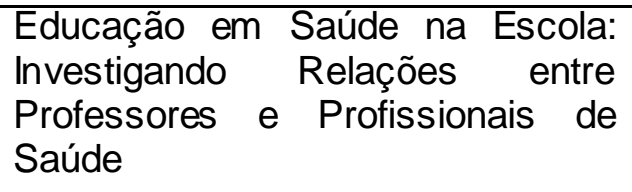 & Venturi, Tiago & 2013 & $\begin{array}{l}\text { D- Universidade } \\
\text { Federal de Santa } \\
\text { Catarina - UFSC }\end{array}$ \\
\hline P25 & $\begin{array}{l}\text { Temas Transversais no Ensino } \\
\text { Fundamental: Educação para a } \\
\text { Saúde e Orientação Sexual }\end{array}$ & $\begin{array}{ll}\text { Zarth, } & \text { Silvana } \\
\text { Maria } & \end{array}$ & 2013 & T-PUC/RGS \\
\hline P26 & $\begin{array}{l}\text { Estilos e Coletivos de Pensamento } \\
\text { das Pesquisas de Educação em } \\
\text { Saúde na Escola (2005 a 2015) }\end{array}$ & $\begin{array}{l}\text { Schwingel, } \\
\text { Tatiane Cristina } \\
\text { Possel Greter }\end{array}$ & 2016 & $\begin{array}{l}\text { D - Universidade } \\
\text { Regional do } \\
\text { Noroeste do } \\
\text { Estado do Rio } \\
\text { Grande do Sul - }\end{array}$ \\
\hline
\end{tabular}


Bio - grafía. Escritos sobre la Biología y su Enseñanza. ISSN 2027-1034

Edición Extraordinaria. p.p. 1360 - 1368

Memorias del IX Encuentro Nacional de Experiencias en Enseñanza de la Biología y la Educación Ambiental. IV Congreso Nacional de Investigación en Enseñanza de la Biología.

\begin{tabular}{|l|l|l|l|l|}
\hline & & & & UNIJUI \\
\hline
\end{tabular}

Fonte: Autoras, 2016.

\section{APRESENTAÇÃO E DISCUSSÃO DOS RESULTADOS}

A análise dos dados possibilitou reconhecer seis categorias: Educação e Sexualidade; Educação em Saúde e o Processo Formativo de Professores; Saúde e Educação; Educação e Hábitos Alimentares; Educação em Saúde nos Livros Didáticos; Educação e Promoção da Saúde.

Na categoria Educação e Sexualidade, nove investigações (P02, P07, P08, P09, P18, P04, P05, P17 e P25) são resultantes de projetos, estudos de caso e desenvolvimento de oficinas com alunos e professores sobre sexualidade na escola. Os pesquisadores citam que o conhecimento sobre educação sexual e sexualidade de professores e alunos continua apresentado numa visão preventiva, com muitos tabus e mitos sobre o tema. Defendem que as escolas devem trabalhar na perspectiva da educação e da sexualidade com discussões da dimensão biológica, psicológica, cultural, comportamental, etc... Portanto, é fundamental investir em formações, problematizações, no trabalho coletivo e diálogos para promover entendimento desse tema. Acerca desse tópico, as investigações realizadas por Vilaça ${ }^{3}$ (2017), em Portugal, apontam para a necessidade de um trabalho colaborativo entre escolas, supervisão pedagógica e universidade com objetivo de promover e avançar nos entendimentos da ES e da sexualidade, a partir das questões e reflexões sobre gênero, diversidade sexual e relações interpessoais. O foco é o desenvolvimento de projetos orientados para resolução de problemas reais pela via da transformação social, a partir do ensino.

As discussões e reflexões sobre a ES e Processo Formativo de Professores (P13, P14, P21, P23, P24) revelam a ausência de um trabalho formativo, que invista em outra perspectiva de Educação em Saúde na escola. Os autores evidenciam a formação centralizada nas questões biológicas, de identificação das causas das doenças e sua eliminação com pouca ênfase na promoção da qualidade de vida dos sujeitos. Carvalho \& Jourdan (2014) e Vilaça (2017) afirmam que há necessidade de promover a responsabilidade pela própria saúde e pela saúde dos outros, permitindo a cada um perceber criticamente cada situação real, para adotar o comportamento mais adequado e eficiente a fim de promover uma vida de qualidade. A formação de professores e as intervenções nas escolas, a partir de projetos, oficinas, entre outros é o caminho para o desenvolvimento de outra forma de pensar a saúde e a ES portanto, a necessidade de continuar investindo e pesquisando os temas correlatos a saúde, a sexualidade, a alimentação e os materiais (para)didáticos afim de pensar e promover um alargamento desse conceito que é tão caro ao Ensino.

As questões do ensino da saúde foram agrupadas na categoria Saúde e Educação (P06, P11, P22). Os três documentos analisados evidenciam os conhecimentos de professores e alunos da Educação Básica acerca da tuberculose e da dengue. Afirmam, ainda, que os textos de divulgação científica possibilitam o

\footnotetext{
${ }^{3}$ Entrevista concedida a uma das autoras no Instituto de Educação da Universidade do Minho,

Braga - Portugal, em abril de 2017.
} 
Bio - grafía. Escritos sobre la Biología y su Enseñanza. ISSN 2027-1034

Edición Extraordinaria. p.p. 1360 - 1368

Memorias del IX Encuentro Nacional de Experiencias en Enseñanza de la Biología y la Educación Ambiental. IV Congreso Nacional de Investigación en Enseñanza de la Biología.

ensino de ES. Dois estudos apontam que o conceito biomédico de saúde se sobrepõe ao entendimento dos sujeitos. Os três autores defendem a discussão de temas relacionados à educação em saúde como campo profícuo para ações e trabalho pedagógico nessa área.

Na categoria Educação e Hábitos Alimentares (P10, P12), a promoção da saúde pela alimentação saudável e conscientização dos benefícios de uma mudança de padrão alimentar na escola e na vida dos sujeitos foram tematizados. Sob esse viés, Loureiro (2004), expressa que educar para comer bem é um desafio. A mudança de comportamentos alimentares deve ser focalizada mais em melhorar o nível de saúde do que na redução de riscos, considerando comportamentos positivos por meio da aquisição de conhecimentos e de atitudes gerais para se cuidar. "A obtenção de comportamentos específicos e a melhoria da capacidade de fazer escolhas saudáveis dependem de uma genuína vontade pessoal, das competências adquiridas e do sentimento de auto-eficácia" (Loureiro, 2004, p. 45).

Educação e Saúde nos Livros Didáticos (LD) foi tratada nos documentos P16 e P20 pela análise da saúde nas imagens e textos. Os autores apontam que nos livros há diferentes abordagens de ES: biomédica, comportamental ou socioambiental conforme Martins, Santos \& El-Hani, (2012) e Westphal (2006), com predominância dos modelos biomédico e comportamental. P16 sinaliza a produção de materiais paradidáticos com outra abordagem de saúde como caminho no ensino da ES. Pois, como P20 expressa em sua análise, a abordagem das viroses nos livros didáticos apresentou uma concentração de informações "nos aspectos biológicos dos vírus sendo, portanto, insuficientes quando se almeja ações de educação para a saúde"(P20, 2012, p.07).

Os textos da Educação e Promoção da Saúde (P03, P19) propõem tessituras que conduzem a população a um estado de saúde. As pesquisas realizadas na escola apresentam uma visão de saúde curativa, longe do que é esperado na promoção da saúde. De acordo com os autores, os sujeitos investigados mantêm uma visão ingênua das questões de saúde, o que os afasta de uma condição de sujeitos críticos com direitos e escolhas autônomas. Esse cenário é decorrente da falta de formação que aborde esta perspectiva, políticas públicas mais eficazes e planejamento conjunto entre escolas e demais segmentos da sociedade como expressa o seguinte excerto "não paramos para refletir sobre nossas ações, não nos planejamos, não questionamos o que nos é imposto, apenas repetimos e massificamos, tornando nossos educandos dependentes na escola, ou nas unidades de saúde" (P19, 2012, p. 75).

O P26 investigou a educação em saúde no âmbito escolar, buscando estabelecer os estilos e coletivos de pensamento das pesquisas em Saúde na Escola (2005 a 2015), e P01 explorou a pedagogia da Educação em Saúde Coletiva como potência de composição de cenários de ensino e aprendizagem na formação de sanitaristas. Estas problematizações excluíram os trabalhos das categorias supracitadas.

A análise das dissertações e teses permitiu identificar questões como aquelas relativas a formação dos licenciandos de Ciências Biológicas, apontando aspectos que devem ser considerados pelos cursos de formação de professores. Questões que perpassam a análise e produção de material didático com outra perspectiva de saúde, 
Bio - grafía. Escritos sobre la Biología y su Enseñanza. ISSN 2027-1034

Edición Extraordinaria. p.p. 1360 - 1368

Memorias del IX Encuentro Nacional de Experiencias en Enseñanza de la Biología y la Educación Ambiental. IV Congreso Nacional de Investigación en Enseñanza de la Biología.

assim como o desenvolvimento de um ensino tanto na universidade como na escola que contemple o debate e a reflexão de temas emergentes como a educação em sexualidade pautada na defesa da felicidade e dignidade humana, a alimentação saudável, a sustentabilidade e o ambiente, a drogatização pelo viés da promoção da saúde e do bem-estar. É essencial a realização de uma prática pedagógica que vá além da abordagem biológica, no sentido de propiciar um ensino para o desenvolvimento da cidadania e de uma aprendizagem significativa fundamentadas sócio-cultural, ética e política da realidade.

\section{CONSIDERAÇÕES FINAIS}

A partir do estado do conhecimento da produção acadêmica de Saúde e Educação em Saúde, estabeleceu-se um panorâma do que está sendo produzido em termos de dissertações e teses brasileiras, nos últimos anos, sobre esse assunto. Identifica-se uma preocupação da comunidade acadêmica em compreender e desenvolver ações e práticas pedagógicas que promovam 0 alargamento do entendimento da ES, na perspectiva da promoção e da qualidade de vida. Os pesquisadores são unânimes ao dizer que os professores têm sua formação na área da saúde embasada no modelo biomédico, enfatizando os aspectos fisiológicos dos processos de saúde-doença, fator esse que se reflete em sua prática de sala de aula. Em suma, para avançar na compreensão da ES que promova o bem-estar, é necessário investir em formação permanente dos professores e desenvolver novas práticas e investigações em parcerias colaborativas entre escola e universidade.

\section{BIBLIOGRAFÍA}

Carvalho, G. S. \& Jourdan, D. ( 2014). Literacia em Saúde na Escola: a importância dos contextos sociais. In: Júnior, C. A. O. M; JúNIOR, A. L; CORAZZA, M. A. (Eds), Ensino de Ciências: múltiplas perspectivas, diferentes olhares. Curitiba, PR: CRV.

Lüdke, M. \& André, M. E. D. A. (2013). Pesquisa em educação: abordagens qualitativas. São Paulo: EPU.

Martins, L.; Santos, G. S.; El-Hani, C. N. (2012). Abordagens de saúde em um livro didático de biologia largamente utilizado no ensino médio brasileiro. Investigações em Ensino de Ciências, POA, 17, 1, 249-283.

Mohr, A. (2002). A natureza da educação e saúde no Ensino Fundamental e os professores de ciências. Dissertação de doutoramento, Universidade Federal de Santa Catarina, Florianópolis, Santa Catarina.

Monteiro, P. H. N; Bizzo, N. (2015). A saúde na escola: análise dos documentos de referência nos quarenta anos de obrigatoriedade dos programas de saúde, 1971-2011. História, Ciências, Saúde - Manguinhos, RJ, 22, 2, 411-427.

Morosini, M. C. (2015). Estado de conhecimento e questões do campo científico. Educação, Santa Maria, 40, 1, 101-116. 
Bio - grafía. Escritos sobre la Biología y su Enseñanza. ISSN 2027-1034

Edición Extraordinaria. p.p. 1360 - 1368

Memorias del IX Encuentro Nacional de Experiencias en Enseñanza de la Biología y la Educación Ambiental. IV Congreso Nacional de Investigación en Enseñanza de la Biología.

Westphal, M. F. (2006). Promoção da Saúde e Prevenção de Doenças. In: Campos, G. W. de S. et al (Eds), Tratado de Saúde Coletiva. SP-RJ: HUCITEC -FIOCRUZ, 635667. 